\title{
Approximation by pseudo-linear operators
}

\author{
Barnabás Bede ${ }^{*}$, Hajime Nobuhara ${ }^{2}$, Martina Daňkováa ${ }^{3}$ \\ Antonio Di Nola ${ }^{4}$ \\ ${ }^{1}$ Department of Mathematics, The University of Texas- Pan American, Edinburg, \\ TX 78541, USA \\ ${ }^{2}$ Department of Intelligent Interaction Technologies, Graduate School of Systems \\ and Information Engineering, University of Tsukuba Tenoudai 1-1-1, Tsukuba \\ science city, Ibaraki 305-8573, Japan \\ ${ }^{3}$ Institute for Research and Applications of Fuzzy Modeling, University of Ostrava \\ 30. Dubna 22, 70103 Ostrava, Czech Republic \\ ${ }^{4}$ Dipartamento di Matematica e Informatica, Universitá di Salerno, Via S. \\ Allende, 84081 Baronissi (Salerno), Italy,
}

\begin{abstract}
The approximation operators provided by classical Approximation Theory use exclusively as underlying algebraic structure the linear structure of the reals. Also they are all linear operators. We address in the present paper the following problems: Need all the approximation operators be linear? Is the linear structure the only one which allows us to construct particular approximation operators? As an answer to this problem we propose new, particular, pseudo-linear approximation operators, which are defined in some ordered semirings. We study these approximations from a theoretical point of view and we obtain that these operators have very similar properties to those provided by classical Approximation Theory. In this sense we obtain Uniform Approximation Theorems of Weierstrass type, and Jackson-type error estimates in approximation by these operators.
\end{abstract}

Key words: approximation, pseudo-analysis, Shepard operator, nonlinear approximation operator, pseudo-linear approximation

\footnotetext{
* Corresponding author

Email address: bedeb@utpa.edu, bede.barna@bgk.bmf.hu ( Barnabás Bede ${ }^{1}$ ).
} 


\section{Introduction}

In classical Functional Analysis, the underlying algebraic structure is the linear space structure and the same is true for the classical Approximation Theory. In several research fields including Decision Analysis, Fuzzy Set Theory, Operations Research, Automatic Control and Mathematical Physics, idempotent analysis and the pseudo-linear structure have already shown their power in problems where the linear structure is not helpful (see e.g. [11], [20], [8]). The pseudo-linear structure is that of an ordered semiring or a dioïd (if the canonical preorder is an order relation), usually on a subset of the real numbers, [8]. The mathematical analysis over this structure is called idempotent analysis, [11] or pseudo-analysis [19], [17].

We address in the present paper the same problem for Approximation Theory i.e., is the linear structure the only one that can be used in classical Approximation Theory? Need all the approximation operators be linear?

In the literature there are several attempts to concerned with approximation on algebraic structures different from the field of reals. Let us mention here the work [14], where M. Minoux addressed the problem of optimal segmentation (see also [7]), for finding best possible piecewise representation of a function in a single variable. Also, in [15], an integral-form nonlinear approximation operator is considered in some modular spaces.

Here we continue this line of research in the setting of classical Approximation Theory and Pseudo-Analysis, by defining particular, Shepard-type, pseudolinear approximation operators in three types of ordered semirings, namely the max-product semiring, the max-min semiring and semirings with generated pseudo-operations.

First we would like to formulate the above proposed problem in a more clear way, but in this case we need a short discussion about classical Approximation Theory.

The main problem solved by classical approximation theory is to approximate a continuous function $f:[a, b] \rightarrow \mathbb{R}$, where $[a, b]$ is a real interval, by some simpler function. Classical approximation theory provides many different approximation operators: Bernstein polynomials, Shepard-Balázs operators, Jackson-type trigonometric polynomials, wavelets (see e.g. [5], [3]), to mention only a few. These approximations are using exclusively the linear structure over $\mathbb{R}$ as underlying algebraic structure. Usually, the form of such an operator is

$$
L_{n}(f, x)=\sum_{i=0}^{n} K_{n}\left(x, x_{i}\right) \cdot f\left(x_{i}\right)
$$


where $x_{i} \in[a, b], i=0, \ldots, n$ are fixed knots and $K_{n}\left(x, x_{i}\right)$ are functions with a relatively simple expression (polynomials, trigonometric polynomials, rational functions, wavelets).

Let us observe that all these operators are linear i.e., $L_{n}(f+g, x)=L_{n}(f, x)+$ $L_{n}(g, x), \forall f, g:[a, b] \rightarrow \mathbb{R}$ continuous.

The main theorems in classical approximation theory are the Weierstrass-type approximation theorems, which state that some function can be uniformly approximated by some approximations, and Jackson-type error estimates which are given in terms of the modulus of continuity and usually these show us that the approximation error is at most of the order $O\left(\omega\left(f, \frac{1}{n}\right)\right)$.

It is now natural to formulate and study the problem: whether we can change the underlying algebraic structure from the field of reals to some more general structures? Also, which important properties will still hold true in the new setting?

In this sense we presented in the recent paper [4], max-product Shepard approximations. For these approximations we have obtained Weierstrass-type uniform approximation theorem and for the approximation error we obtained Jackson-type estimates.

The idea and the problem came from both the theory and applications of Fuzzy Sets. In several papers, (see e.g. [21], [22], [2], [10], [28]) the approximation capability of fuzzy systems is studied. Also, in several image processing applications, different operations are used (see e.g. [16], [9]). These lead us to the idea of defining and studying pseudo-linear approximation operators. Also, the algebraic structure of an ordered semiring that we are using is closely connected to the Fuzzy Set Theory, as it is shown in the very recent paper [8].

In the present paper we discuss in detail the problem of how approximations can be defined based on different types of ordered semirings. The possibility to change the algebraic structure from the classical linear structure to other, more general structures, allows us to generalize several important results of classical Approximation Theory in a new direction.

So, the purpose of the present paper is to approximate a continuous function $f: X \rightarrow[0, \infty)$, where $(X, d)$ is a compact metric space, by approximations defined on algebraic structures different from the field of real numbers. We will use three types of ordered semirings: the max-product semiring of positive real numbers, max-min semiring on the $[0,1]$ interval and semirings with generated pseudo-operations over the set of positive real numbers. The later class is an isomorphic image of the semiring of positive reals, and moreover, it contains as a particular case the linear structure, so we have a generalization of the classical Shepard-Balázs operator. The purpose of this paper is an analysis of 
the theoretical aspects of the problem and future work will contain practical results and we will show that in several applications, the new pseudo-linear approximations outperform their classical counterparts. Let us also mention that the present paper opens new research direction, the topic of pseudo-linear approximation.

Let us remark that in classical approximation theory the term "nonlinear approximation" is used in a quite different sense (it is approximation by using a dictionary of functions, see e.g. [6]).

Let us also remark here that in the proofs we use not only the ordered semiring structure (recall that we deal with subsets of the real line so whenever needed, we can use the classical operations as well). The metric structure considered here is the Euclidean distance between the reals, but also, in Section 4, we will use a generalized metric space structure (see [23], [29], [30]).

After a preliminary section where we discuss the three types of ordered semirings used in this paper and some analytic properties, we recall in Section 3 the max-product approximations. Max-min approximations are introduced in Section 4. Section 5 concerns pseudo-linear approximation operators based on an ordered semiring structure with generated pseudo-operations.

\section{Preliminaries}

\subsection{Ordered semirings}

Let $I$ be a closed or semiclosed subinterval of $[0, \infty]$. The operation $\oplus: I \times I \rightarrow$ $I$ is called a pseudo-addition if it is commutative, nondecreasing, associative and has a zero element denoted by $\mathbf{0}$.

The operation $\odot: I \times I \rightarrow I$ is a pseudo-multiplication if it is commutative, nondecreasing, associative and for which there exists a unit element $\mathbf{1} \in I$. Let us further suppose that $\odot$ is distributive with respect to $\oplus$ i.e.,

$$
x \odot(y \oplus z)=x \odot y \oplus x \odot z .
$$

for any $x, y, z \in I$.

We will consider in the examples presented in this paper the following 3 cases:

Case 1. $I=[0, \infty), \oplus=\vee$ and $\odot=\cdot$, that is the max-product semiring of positive reals. 
Case 2. $I=[0,1], \oplus=\vee$ and $\odot=\wedge$, that is the max-min semiring over the $[0,1]$ interval, i.e., the max-min fuzzy algebra. We observe that $\vee$ and $\wedge$ operations can be extended over $[0, \infty]$ interval but in this case the unit is $\infty$. Later, in the proofs of some theorems we will use the extended operations (in these parts we will not use the properties related to the unit).

Case 3. Semirings with pseudo-operations defined by a monotone and continuous generator $g:[0, \infty) \rightarrow[0, \infty)$ (see [1], [13], [19], [18], [8]). Moreover, from this class we consider only strict pseudo-additions, that is $\oplus$ is strictly increasing on $(0, \infty) \times(0, \infty)$. In this case by Aczél's representation theorem there exists a monotone function $g:[0, \infty) \rightarrow[0, \infty)$ such that $g(0)=0$ and

$$
x \oplus y=g^{-1}(g(x)+g(y)) .
$$

In this case the unique pseudo-multiplication associated to $\oplus$ is given by

$$
x \odot y=g^{-1}(g(x) \cdot g(y)) .
$$

It is easy to check that $\mathbf{1}=g^{-1}(1)$ is neutral element with respect to $\odot$ in the sense that $x \odot \mathbf{1}=x$ for any $x \in(0, \infty)$. By convention, the operation $\odot$ will have in the present paper always higher priority than the operation $\oplus$. In this last case we have an isomorphic copy of the semiring of positive real numbers with the usual operations. The algebraic structure obtained here is not a general fuzzy algebra. This can be seen easily from the fact that in our case the distributivity law holds, and this is not generally the case in fuzzy algebras.

However this later case is isomorphic to the semiring of positive reals with the usual addition and multiplication, the approximations presented later in this paper are not obtained as a copy by the isomorphism of their classical counterpart. Instead we define and study these approximations inside the ordered semiring structure obtained in this case.

The algebraic structure induced by the above described pseudo-operations, in all the presented cases is that of an ordered semiring and moreover the order relation is compatible with the operations considered. Such algebraic structures, despite the apparent differences from fuzzy algebra are strongly connected to Fuzzy Sets Theory, as it is shown in e.g., [8].

\subsection{Some analytic properties}

Usually, the error estimates in classical approximation theory are provided in terms of the modulus of continuity. So, let us recall its definition and main properties adapted to our case (for the general definition see [3]). 
Definition 1 Let $(X, d)$ be a compact metric space and $([0, \infty),|\cdot|)$ the metric space of positive reals endowed with the usual Euclidean distance. Let $f: X \rightarrow$ $[0, \infty)$ be bounded. Then the function $\omega(f, \cdot):[0, \infty) \rightarrow[0, \infty)$, defined by

$$
\omega(f, \delta)=\bigvee\{|f(x)-f(y)| ; x, y \in X, d(x, y) \leq \delta\}
$$

is called the modulus of continuity of $f$.

Theorem 2 The following properties hold true

i) $|f(x)-f(y)| \leq \omega(f, d(x, y))$ for any $x, y \in X$;

ii) $\omega(f, \delta)$ is nondecreasing in $\delta$;

iii) $\omega(f, 0)=0$;

iv) $\omega\left(f, \delta_{1}+\delta_{2}\right) \leq \omega\left(f, \delta_{1}\right)+\omega\left(f, \delta_{2}\right)$ for any $\delta_{1}, \delta_{2}, \in[0, \infty)$;

v) $\omega(f, n \delta) \leq n \omega(f, \delta)$ for any $\delta \in[0, \infty)$ and $n \in \mathbb{N}$;

vi) $\omega(f, \lambda \delta) \leq(\lambda+1) \cdot \omega(f, \delta)$ for any $\delta, \lambda \in[0, \infty)$;

vii) $f$ is continuous if and only if $\lim _{\delta \rightarrow 0} \omega(f, \delta)=0$.

Since the approximation operators defined later in this paper are often said to be of Shepard-type, let us recall the definition and approximation properties of the so-called Shepard-Balázs (sometimes called only Shepard) operators (see [24], [26], [25], [32], [12], [28]). We have chosen the Shepard kernel in the investigations presented here for its simplicity. For future research one can use as well other kernels.

Let $f:[0,1] \rightarrow \mathbb{R}$ be a continuous function and $x_{i} \in[0,1], i \in\{0, \ldots, n\}$, $n \geq 1$ be equally spaced points and $\lambda>2$. Let also $B_{n}^{\lambda}\left(x, x_{i}\right)=\frac{\frac{1}{\left|x-x_{i}\right|^{\lambda}}}{\sum_{i=0}^{n} \frac{1}{\left|x-x_{i}\right|^{\lambda}}}$, if $x \in[0,1] \backslash\left\{x_{0}, x_{1}, \ldots, x_{n}\right\}$ and $B_{n}^{\lambda}\left(x_{j}, x_{i}\right)=\delta_{i j}, i, j \in\{0, \ldots, n\}\left(\delta_{i j}\right.$ is the Kronecker symbol) denote the classical Shepard kernel. Then

$$
S_{n}^{\lambda}(f, x)=\sum_{i=0}^{n} B_{n}^{\lambda}\left(x, x_{i}\right) f\left(x_{i}\right),
$$

is the classical Shepard-Balázs (interpolatory) operator.

Theorem 3 ([26], [27])Let $f:[0,1] \rightarrow \mathbb{R}$ be a continuous function. Then, the Shepard-Balázs operator defined as in (1) satisfies the following Jackson-type error estimate:

$$
\left|S_{n}^{\lambda}(f, x)-f(x)\right| \leq C \omega\left(f, \frac{1}{n}\right)
$$

where $C$ is a constant which does not depend on $n$. 
In order to study approximation properties of the max-product and max-min approximations defined later in this paper we need the following two lemmas.

Lemma 4 For any $a_{i}, b_{i} \in[0, \infty), i \in\{0, \ldots, n\}$ we have

$$
\left|\bigvee_{i=0}^{n} a_{i}-\bigvee_{i=0}^{n} b_{i}\right| \leq \bigvee_{i=0}^{n}\left|a_{i}-b_{i}\right|
$$

PROOF. Since max operation is nondecreasing in its arguments, we have

$$
\bigvee_{i=0}^{n} a_{i}=\bigvee_{i=0}^{n}\left|b_{i}+a_{i}-b_{i}\right| \leq \bigvee_{i=0}^{n} b_{i}+\left|a_{i}-b_{i}\right|
$$

and it follows

$$
\bigvee_{i=0}^{n} a_{i} \leq \bigvee_{i=0}^{n} b_{i}+\bigvee_{i=0}^{n}\left|a_{i}-b_{i}\right|
$$

This inequality together with the symmetric case implies the statement of the lemma.

In the proof of the next Lemma we "go out" from the structure of the maxmin ordered semiring on $[0,1]$ interval and we use the extended operations over $[0, \infty]$ together with the classical addition. This does not lead to incorrect proof since we do not use here the properties of the unit.

Lemma 5 For any $x, y, z \in[0,1]$ we have

$$
|x \wedge y-x \wedge z| \leq x \wedge|y-z|
$$

PROOF. It is easy to see that if we suppose $y>z$, we have

$$
x \wedge y=x \wedge(z+y-z) \leq x \wedge z+x \wedge(y-z),
$$

inequality which together with the symmetric case implies

$$
|x \wedge y-x \wedge z| \leq x \wedge|y-z|
$$

\section{Max-product approximation}

Let us consider a continuous function $f: X \rightarrow[0, \infty),(X, d)$ being a compact metric space and on $[0, \infty)$ we consider the usual Euclidean distance. Let also, 
$x_{i} \in X, i \in\{0, \ldots, n\}, n \geq 1$ be fixed sampled data (i.e. we suppose that the values $f\left(x_{i}\right)$ are known). The idea of defining max-product approximation operators is very simple: we change the algebraic streucture from the field of reals to the ordered, max-product semiring of positive reals.

The general discrete form of a max-product approximation is given by

$$
P_{n}(f, x)=\bigvee_{i=0}^{n} K_{n}\left(x, x_{i}\right) \cdot f\left(x_{i}\right)
$$

where $K_{n}\left(\cdot, x_{i}\right): X \rightarrow[0, \infty), i=0, \ldots, n$ are some given continuous functions. It is easy to check that $P_{n}(f, \cdot): X \rightarrow[0, \infty)$ is a continuous function.

Let us denote by $C(X,[0, \infty))$ the space of continuous functions $f: X \rightarrow$ $[0, \infty)$ endowed with the uniform distance $\|f-g\|=\bigvee_{x \in X}|f(x)-g(x)|$.

Proposition 6 The operator $P_{n}: C(X,[0, \infty)) \rightarrow C(X,[0, \infty)), P_{n}(f, x)=$ $\bigvee_{i=0}^{n} K_{n}\left(x, x_{i}\right) \cdot f\left(x_{i}\right)$, is continuous in the uniform distance and it is pseudo linear in the sense that

$$
P_{n}(\alpha \cdot f \vee \beta \cdot g, x)=\alpha \cdot P_{n}(f, x) \vee \beta \cdot P_{n}(g, x) .
$$

PROOF. We have

$$
\left|P_{n}(f, x)-P_{n}(g, x)\right|=\left|\bigvee_{i=0}^{n} K_{n}\left(x, x_{i}\right) \cdot f\left(x_{i}\right)-\bigvee_{i=0}^{n} K_{n}\left(x, x_{i}\right) \cdot g\left(x_{i}\right)\right| .
$$

By Lemma 4 we have successively

$$
\begin{aligned}
\left|P_{n}(f, x)-P_{n}(g, x)\right| & \leq \bigvee_{i=0}^{n}\left|K_{n}\left(x, x_{i}\right) \cdot f\left(x_{i}\right)-K_{n}\left(x, x_{i}\right) \cdot g\left(x_{i}\right)\right| \\
& \leq \bigvee_{i=0}^{n} K_{n}\left(x, x_{i}\right)\left|f\left(x_{i}\right)-g\left(x_{i}\right)\right| \\
& \leq \bigvee_{i=0}^{n} K_{n}\left(x, x_{i}\right)\|f-g\| .
\end{aligned}
$$

Since $K_{n}\left(\cdot, x_{i}\right)$ are continuous on the compact $X$, they are bounded and we get

$$
\left\|P_{n}(f)-P_{n}(g)\right\| \leq M\|f-g\|
$$

where $M=\bigvee_{i=0}^{n} \bigvee_{x \in X} K_{n}\left(x, x_{i}\right)$. The pseudo-linearity of $P_{n}$ is obvious. 
An example of such an operator can be found in [4], where max-product Shepard operator is defined and studied. We define the maxitive Shepard kernel as follows:

$$
K_{n, \lambda}\left(x, x_{i}\right)=\frac{\frac{1}{d\left(x, x_{i}\right)^{\lambda}}}{\bigvee_{j=0}^{n} \frac{1}{d\left(x, x_{j}\right)^{\lambda}}}, \text { if } x \notin\left\{x_{0}, \ldots x_{n}\right\}
$$

and $K_{n, \lambda}\left(x_{j}, x_{i}\right)=\delta_{i j}, i, j=0, \ldots, n, n \geq 1$, and $\lambda \geq 1$ is a constant. It is easy to check that $K_{n, \lambda}\left(\cdot, x_{i}\right), i=0, \ldots, n$ are continuous. Then the max-product Shepard operator can be written

$$
S h_{n}^{\lambda}(f, x)=\bigvee_{i=0}^{n} K_{n, \lambda}\left(x, x_{i}\right) \cdot f\left(x_{i}\right)=\frac{\bigvee_{i=0}^{n} \frac{f\left(x_{i}\right)}{d\left(x, x_{i}\right)^{\lambda}}}{\bigvee_{j=0}^{n} \frac{1}{d\left(x, x_{j}\right)^{\lambda}}}, \text { if } x \notin\left\{x_{i}: i=0, \ldots, n\right\}
$$

and $S h_{n}^{\lambda}\left(f, x_{i}\right)=f\left(x_{i}\right)$ for $i=0, \ldots, n$. It is easy to check that $S h_{n}^{\lambda}(f, \cdot)$ is continuous since $K_{n, \lambda}\left(\cdot, x_{i}\right)$ are continuous.

The following approximation properties were obtained in [4].

Theorem 7 Let $f: X \rightarrow[0, \infty)$, be continuous, $x_{i} \in X, i \in\{0, \ldots, n\}$ be fixed sampled data and $S h_{n}^{\lambda}(f, x)$ as in (4), $\lambda \geq 1$. Then the following error estimate holds true

$$
\left|S h_{n}^{\lambda}(f, x)-f(x)\right| \leq\left(m \bigwedge_{i=0}^{n} d\left(x, x_{i}\right)+1\right) \cdot \omega\left(f, \frac{1}{m}\right),
$$

for any $x \in X$ and any $m \in \mathbb{N}$.

Moreover, for any $\varepsilon>0$ there exists $n \in \mathbb{N}$ and a sequence of points $x_{i}$, $i \in\{0, \ldots, n\}$ such that for $S_{n}^{\lambda}(f, x)$ defined by using these points we have $\left|S h_{n}^{\lambda}(f, x)-f(x)\right|<\varepsilon$, for all $x \in X$.

Particularly simple error estimate can be obtained in the case of equally spaced data in the unit interval.

Theorem 8 Let $f:[0,1] \rightarrow[0, \infty)$ be continuous, $x_{i}=\frac{i}{n}, i=0, \ldots, n, n \geq 1$, $\lambda \geq 1$. Then for the error of approximation of $f$ by $S h_{n}^{\lambda}(f, x)$ given as in (4) we have

$$
\left|S h_{n}^{\lambda}(f, x)-f(x)\right| \leq \frac{3}{2} \omega\left(f, \frac{1}{n}\right), \forall x \in[0,1]
$$

Here let us remark that in the theory of classical Shepard-Balázs operators, it is proved that for $\lambda>2$ we have the Jackson-type estimate, however, for 
$1 \leq \lambda \leq 2$, we have weaker error estimates (see [33], [28]). So, at this point max-product approximation "outperforms" classical approximation theory.

\section{Max-min approximation}

In this section we consider the max-min fuzzy algebra over the $[0,1]$ interval. The general form of a max-min approximation operator is similar to the previous max-product case.

Let $(X, d)$ be a compact metric space and $f: X \rightarrow[0,1]$ be a continuous function. Let $x_{i} \in X, f\left(x_{i}\right) \in[0,1]$ be sampled data, $i=0,1, \ldots, n, n \geq 1$. The general discrete form of a max-min approximation operator is

$$
P_{n}(f, x)=\bigvee_{i=0}^{n} K_{n}\left(x, x_{i}\right) \wedge f\left(x_{i}\right)
$$

where $K_{n}\left(\cdot, x_{i}\right): X \rightarrow[0,1], i=0, \ldots, n$ are some given continuous functions. It is easy to check that $P_{n}(f, \cdot): X \rightarrow[0,1]$ is a continuous function.

Let us denote by $C(X,[0,1])$ the space of continuous functions $f: X \rightarrow[0,1]$ endowed with the uniform distance.

Proposition 9 The operator $P_{n}: C(X,[0,1]) \rightarrow C(X,[0,1]), P_{n}(f, x)=$ $\bigvee_{i=0}^{n} K_{n}\left(x, x_{i}\right) \wedge f\left(x_{i}\right)$, is continuous and pseudo-linear, in the sense that

$$
P_{n}((\alpha \wedge f) \vee(\beta \wedge g), x)=\left(\alpha \wedge P_{n}(f, x)\right) \vee\left(\beta \wedge P_{n}(g, x)\right)
$$

PROOF. We have

$$
\left|P_{n}(f, x)-P_{n}(g, x)\right|=\left|\bigvee_{i=0}^{n} K_{n}\left(x, x_{i}\right) \wedge f\left(x_{i}\right)-\bigvee_{i=0}^{n} K_{n}\left(x, x_{i}\right) \wedge g\left(x_{i}\right)\right| .
$$

By Lemma 4 and Lemma 5 we have

$$
\left|P_{n}(f, x)-P_{n}(g, x)\right| \leq \bigvee_{i=0}^{n} K_{n}\left(x, x_{i}\right) \wedge\|f-g\|
$$

Since $K_{n}\left(\cdot, x_{i}\right)$ are continuous on the compact $X$, they are bounded and we get

$$
\left\|P_{n}(f)-P_{n}(g)\right\| \leq M\|f-g\|,
$$

where $M=\bigvee_{i=0}^{n} \bigvee_{x \in X} K_{n}\left(x, x_{i}\right)$. The pseudo-linearity of $P_{n}$ is obvious. 
We define in what follows the max-min Shepard operators. Let $(X, d)$ be a compact metric space and $f: X \rightarrow[0,1]$ be a continuous function. Let $x_{i} \in X$, $f\left(x_{i}\right) \in[0,1]$ be fixed sampled data, $i=0,1, \ldots, n, n \geq 1$ and $\lambda \geq 1$ a constant. We consider the maxitive Shepard kernel defined in (3). Then the function

$S h_{n}^{\lambda}(f, x)=\bigvee_{i=0}^{n} K_{n, \lambda}\left(x, x_{i}\right) \wedge f\left(x_{i}\right)=\bigvee_{i=0}^{n}\left(\frac{\frac{1}{d\left(x, x_{i}\right)^{\lambda}}}{\bigvee_{j=0}^{n} \frac{1}{d\left(x, x_{j}\right)^{\lambda}}} \wedge f\left(x_{i}\right)\right)$, for $x \notin\left\{x_{0}, \ldots, x_{n}\right\}$

and $S h_{n}^{\lambda}\left(f, x_{i}\right)=f\left(x_{i}\right), i=0, \ldots, n$ is called the max-min Shepard operator. It is easy to check that $S h_{n}^{\lambda}(f, \cdot)$ is a continuous function (since the maxitive Shepard kernel is continuous).

The following theorem gives an approximation property of max-min Shepard operator.

Theorem 10 Any continuous function $f: X \rightarrow[0,1]$ can be arbitrarily closely approximated by max-min Shepard-type operators, i.e. for any $\varepsilon>0$, there exist $n \in \mathbb{N}$, a sequence of points $x_{i} \in X, i \in\{0, \ldots, n\}$ and $\lambda \geq 1$, such that $\left|S h_{n}^{\lambda}(f, x)-f(x)\right|<\varepsilon$, for all $x \in X$, where $S h_{n}^{\lambda}(f, x)$ is given by (7).

PROOF. It is well known that every compact metric space is totally bounded, i.e. for every $\varepsilon>0$ there exists a finite covering of $X$ by open balls having the radius $\varepsilon$ i.e., $\cup_{k=0}^{n} B_{k}=X, B_{k}$ is an open ball of radius $\varepsilon$ and center $x_{k}$. Let $\varepsilon=\frac{1}{m}, m \in \mathbb{N}$. Then it is easy to see that $\bigwedge_{i=0}^{n} d\left(x, x_{i}\right)<\frac{1}{m}$ for any $x \in X$, which gives $\frac{1}{n}>m$. Let $x$ be an arbitrary fixed element from $X$ and $I_{k}$ be index sets defined by

$$
\bigwedge_{i=0} d\left(x, x_{i}\right)
$$

$$
I_{k}=\left\{i \in\{0, \ldots, n\}: d\left(x, x_{i}\right)<\frac{1}{k}\right\}, k \in\{1,2, \ldots\}
$$

By using Lemma 4 and 5 we have

$$
\left|S h_{n}^{\lambda}(f, x)-f(x)\right| \leq \bigvee_{i=0}^{n}\left(\frac{\frac{1}{d\left(x, x_{i}\right)^{\lambda}}}{\bigvee_{j=0}^{n} \frac{1}{d\left(x, x_{j}\right)^{\lambda}}} \wedge\left|f(x)-f\left(x_{i}\right)\right|\right)
$$


and by Theorem 2, (i) we obtain

$$
\left|S h_{n}^{\lambda}(f, x)-f(x)\right| \leq \bigvee_{i=0}^{n}\left(\frac{\frac{1}{d\left(x, x_{i}\right)^{\lambda}}}{\bigvee_{j=0}^{n} \frac{1}{d\left(x, x_{j}\right)^{\lambda}}} \wedge \omega\left(f, d\left(x, x_{i}\right)\right)\right)
$$

Now, it is easy to check that for any $a_{i}, b_{i} \in[0,1], i=0, \ldots, n$ the inequality

$$
\bigvee_{i=0}^{n} a_{i} \wedge b_{i} \leq \bigvee_{i \in J} a_{i} \vee \bigvee_{i \notin J} b_{i}
$$

holds true for any index set $J \subset\{0, \ldots, n\}$. Then we have

$$
\left|S h_{n}^{\lambda}(f, x)-f(x)\right| \leq \bigvee_{i \in I_{k}} \omega\left(f, d\left(x, x_{i}\right)\right) \vee \bigvee_{i \notin I_{k}} \frac{\frac{1}{d\left(x, x_{i}\right)^{\lambda}}}{\bigwedge_{j=0}^{n} d\left(x, x_{j}\right)^{\lambda}}
$$

where $I_{k}$ is given by (8) and it follows

$$
\begin{aligned}
\left|S h_{n}^{\lambda}(f, x)-f(x)\right| & \leq \omega\left(f, \frac{1}{k}\right) \vee \bigvee_{i \notin I_{k}} \frac{\frac{1}{d\left(x, x_{i}\right)^{\lambda}}}{m^{\lambda}} \\
& =\omega\left(f, \frac{1}{k}\right) \vee \frac{\bigwedge_{i \notin I_{k} d\left(x, x_{i}\right)^{\lambda}}}{m^{\lambda}} .
\end{aligned}
$$

Since for $i \notin I_{k}$ we have $d\left(x, x_{i}\right)>\frac{1}{k}$, we obtain

$$
\left|S h_{n}^{\lambda}(f, x)-f(x)\right| \leq \omega\left(f, \frac{1}{k}\right) \vee \frac{k^{\lambda}}{m^{\lambda}} .
$$

Now let $k=\frac{m}{2}$ then we get

$$
\left|S h_{n}^{\lambda}(f, x)-f(x)\right| \leq \omega\left(f, \frac{2}{m}\right) \vee \frac{1}{2^{\lambda}}
$$

Since for any $\varepsilon>0$ we can take $m$ such that $\omega\left(f, \frac{2}{m}\right)<\varepsilon$ and $\lambda$ such that $\frac{1}{2^{\lambda}}<\varepsilon$, the statement of the theorem is proved.

Particularly simple error estimate can be obtained if we consider equidistant data in the unit interval. Let us remark that here we do not obtain a classical Jackson-type estimate, but a very similar one. 
Theorem 11 Let $f:[0,1] \rightarrow[0,1]$ be continuous and $x_{i}=\frac{i}{n}, i=0, \ldots, n$, equally spaced data. For the max-min Shepard-type approximation operator given by (7) we have the following error estimate

$$
\left|S h_{n}^{\lambda}(f, x)-f(x)\right| \leq \omega\left(f, \frac{1}{n}\right) \vee \frac{1}{3^{\lambda}}, \forall x \in[0,1]
$$

PROOF. For the unit interval the maxitive Shepard kernel is

$$
K_{n}^{\lambda}\left(x, x_{i}\right)=\frac{\frac{1}{\left|x-\frac{i}{n}\right|^{\lambda}}}{\bigvee_{k=0}^{n} \frac{1}{\left|x-\frac{k}{n}\right|^{\lambda}}}
$$

Let $x \in\left[\frac{j}{n}, \frac{j+1}{n}\right]$. Then

$$
\bigvee_{k=0}^{n} \frac{1}{\left|x-\frac{k}{n}\right|^{\lambda}}=\frac{1}{\left|x-\frac{j}{n}\right|^{\lambda}} \vee \frac{1}{\left|x-\frac{j+1}{n}\right|^{\lambda}}
$$

Let us suppose that $x \in\left[\frac{j}{n}, \frac{2 j+1}{2 n}\right]$. Then we obtain

$$
\bigvee_{k=0}^{n} \frac{1}{\left|x-\frac{k}{n}\right|^{\lambda}}=\frac{1}{\left|x-\frac{j}{n}\right|^{\lambda}}
$$

In (9) we have

$$
\begin{aligned}
|S h(x)-f(x)| & \leq \bigvee_{i=0}^{n} \frac{\frac{1}{\left|x-\frac{i}{n}\right|^{\lambda}}}{\left|x-\frac{j}{n}\right|^{\lambda}} \wedge\left|f(x)-f\left(\frac{i}{n}\right)\right| \\
& \leq \bigvee_{i=j}^{j+1}\left|f(x)-f\left(\frac{i}{n}\right)\right| \vee \bigvee_{i \neq j, j+1} \frac{\frac{1}{\left|x-\frac{i}{n}\right|^{\lambda}}}{\frac{1}{\left|x-\frac{j}{n}\right|^{\lambda}}}
\end{aligned}
$$

Then, by the properties of the modulus of continuity we have

$$
\left|S h_{n}^{\lambda}(f, x)-f(x)\right| \leq \omega\left(f,\left|x-\frac{j}{n}\right|\right) \vee \omega\left(f,\left|x-\frac{j+1}{n}\right|\right) \vee \bigvee_{i \neq j, j+1} \frac{\frac{1}{\left|x-\frac{i}{n}\right|^{\lambda}}}{\left|x-\frac{j}{n}\right|^{\lambda}}
$$


By direct computation it follows

$$
\begin{aligned}
\left|S h_{n}^{\lambda}(f, x)-f(x)\right| & \leq \omega\left(f, \frac{1}{n}\right) \vee \frac{\frac{1}{\left|x-\frac{j-1}{n}\right|^{\lambda}}}{\frac{1}{\left|x-\frac{j}{n}\right|^{\lambda}}} \vee \frac{\frac{1}{\left|x-\frac{j+2}{n}\right|^{\lambda}}}{\frac{1}{\left|x-\frac{j}{n}\right|^{\lambda}}} \\
& =\omega\left(f, \frac{1}{n}\right) \vee \frac{\left|x-\frac{j}{n}\right|^{\lambda}}{\left|x-\frac{j-1}{n}\right|^{\lambda}} \vee \frac{\left|x-\frac{j}{n}\right|^{\lambda}}{\left|x-\frac{j+2}{n}\right|^{\lambda}}
\end{aligned}
$$

Let $\varphi:\left[\frac{j}{n}, \frac{2 j+1}{2 n}\right] \rightarrow \mathbb{R}, \varphi(x)=\frac{\left(x-\frac{j}{n}\right)^{\lambda}}{\left(x-\frac{j-1}{n}\right)^{\lambda}}$. Since $\varphi$ is increasing and since we have supposed that $x \in\left[\frac{j}{n}, \frac{2 j+1}{2 n}\right]$, we obtain

$$
\frac{\frac{1}{\left|x-\frac{j-1}{n}\right|^{\lambda}}}{\frac{1}{\left|x-\frac{j}{n}\right|^{\lambda}}}<\frac{\left(\frac{2 j+1}{2 n}-\frac{j}{n}\right)^{\lambda}}{\left(\frac{2 j+1}{2 n}-\frac{j-1}{n}\right)^{\lambda}}=\frac{1}{3^{\lambda}} .
$$

Let $\phi:\left[\frac{j}{n}, \frac{2 j+1}{2 n}\right] \rightarrow \mathbb{R}$, and

$$
\phi(x)=\frac{\left(x-\frac{j}{n}\right)^{\lambda}}{\left(\frac{j+2}{n}-x\right)^{\lambda}} .
$$

Then $\phi(x) \leq \phi\left(\frac{2 j+1}{2 n}\right)=\frac{1}{3^{\lambda}}$. Finally we obtain

$$
\left|S h_{n}^{\lambda}(f, x)-f(x)\right| \leq \omega\left(f, \frac{1}{n}\right) \vee \frac{1}{3^{\lambda}} .
$$

The symmetrical case, (i.e. the case when $x \in\left[\frac{2 j+1}{2 n}, \frac{j+1}{n}\right]$ is similar and the proof is complete.

\section{Approximation based on pseudo-operations given by a monotone and continuous generator}

\subsection{Some properties in generalized metric spaces}

In this section we consider the ordered semiring of positive reals with generated pseudo-operations, defined by a nondecreasing continuous generator $g:[0, \infty) \rightarrow[0, \infty)$ such that $g(0)=0$, as follows:

$$
x \oplus y=g^{-1}(g(x)+g(y))
$$


and

$$
x \odot y=g^{-1}(g(x) \cdot g(y)) .
$$

We denote by $\mathbf{1}=g^{-1}(1)$ the neutral element of the pseudo-multiplication. Also, for simplicity, if $f: X \rightarrow[0, \infty)$ is a function, sometimes we will denote $g \circ f$ by $g f$.

In order to obtain error estimates for the approximation operators defined later in this paper, we need some kind of a "metric structure" over $[0, \infty)$ which is compatible with the above described operations. So, we consider the mapping $d_{g}:[0, \infty)^{2} \rightarrow[0, \infty)$,

$$
d_{g}(x, y)=g^{-1}(|g(x)-g(y)|) \text {. }
$$

Then $d_{g}$ has the following properties (see [23], [29], [30]):

(i) $d_{g}(a, b) \geq 0, \forall a, b \in[0, \infty)$ and $d_{g}(a, b)=0$ if and only if $a=b$;

(ii) $d_{g}(a, b)=d_{g}(b, a), \forall a, b \in[0, \infty)$;

(iii) $d_{g}(a, c) \leq d_{g}(a, b) \oplus d_{g}(b, c), \forall a, b, c \in[0, \infty)$.

These show us that $d_{g}$ has properties similar to the usual Euclidean distance over the reals, except the fact that the triangle inequality is written now in terms of the pseudo-addition. The mapping $d_{g}$ will be called g-distance and let us remark here that $\left([0, \infty), d_{g}\right)$ is a generalized metric space as it is introduced in [23], see also [29].

As in the classical case, one can define the uniform g-distance as $D_{g}\left(f_{1}, f_{2}\right)=$ $\bigvee_{x \in X} d_{g}\left(f_{1}(x), f_{2}(x)\right)$, for any continuous functions $f_{1}, f_{2}: X \rightarrow[0, \infty)$. Let $C(X,[0, \infty))$ be the space of continuous functions $f: X \rightarrow[0, \infty)$ endowed with the uniform g-distance (continuity of $f$ is understood in the Euclidean distance). An operator $P: C(X,[0, \infty)) \rightarrow C(X,[0, \infty))$ is said to be continuous in the uniform g-distance if $\forall \varepsilon>0, \exists \delta>0$ such that $D_{g}\left(P\left(f_{1}\right), P\left(f_{2}\right)\right)<\varepsilon$, if $D_{g}\left(f_{1}, f_{2}\right)<\delta$. Also, if a sequence converges in the uniform g-distance we say that it converges g-uniformly.

Analogously to the classical case in Definition 1, we define the g-modulus of continuity.

Definition 12 Let $(X, d)$ be a compact metric space and $\left([0, \infty), d_{g}\right)$ the space of positive reals endowed with the $g$-distance given in (10). Let $f: X \rightarrow[0, \infty)$ be bounded in the Euclidean distance. Then the function $\omega_{g}(f, \cdot):[0, \infty) \rightarrow$ $[0, \infty)$, defined by

$$
\omega_{g}(f, \delta)=\bigvee\left\{d_{g}(f(x), f(y)) ; x, y \in X, d(x, y) \leq \delta\right\}
$$


is called the g-modulus of continuity of $f$.

Similar to the classical case in Theorem 2, we can obtain several properties for the g-modulus of continuity.

Theorem 13 The following properties hold true

i) $d_{g}(f(x), f(y)) \leq \omega_{g}(f, d(x, y))$ for any $x, y \in X$;

ii) $\omega_{g}(f, \delta)$ is nondecreasing in $\delta$;

iii) $\omega_{g}(f, 0)=0$;

iv) $\omega_{g}\left(f, \delta_{1}+\delta_{2}\right) \leq \omega_{g}\left(f, \delta_{1}\right) \oplus \omega_{g}\left(f, \delta_{2}\right)$ for any $\delta_{1}, \delta_{2}, \in[0, \infty)$;

v) $\omega_{g}(f, n \delta) \leq g^{-1}(n) \odot \omega_{g}(f, \delta)$ for any $\delta \in[0, \infty)$ and $n \in \mathbb{N}$;

vi) $\omega_{g}(f, \lambda \delta) \leq g^{-1}(\lambda+1) \odot \omega_{g}(f, \delta)$ for any $\delta, \lambda \in[0, \infty)$;

vii) $\omega_{g}(f, \delta)=g^{-1}(\omega(g f, \delta))$, for any $\delta$, where $\omega$ is the classical modulus of continuity given by Definition 1.

viii) $f$ is continuous in the Euclidean distance if and only if $\lim _{\delta \rightarrow 0} \omega_{g}(f, \delta)=$ 0 .

PROOF. It is easy to check that properties (i)-(iii) hold true.

(iv) We start from

$$
d_{g}(f(x), f(z)) \leq d_{g}(f(x), f(y)) \oplus d_{g}(f(y), f(z)), x, y, z \in X
$$

Let us suppose that $d(x, y) \leq \delta_{1}$ and $d(y, z) \leq \delta_{2}$. Then $d(x, z) \leq \delta_{1}+\delta_{2}$. Now, if we take into account that $\oplus$ is nondecreasing, in the previous inequality we obtain

$$
\omega_{g}\left(f, \delta_{1}+\delta_{2}\right) \leq \omega_{g}\left(f, \delta_{1}\right) \oplus \omega_{g}\left(f, \delta_{2}\right) .
$$

(v) We use the inequality in (iv) $n$ times and we get

$$
\omega_{g}(f, n \delta) \leq \bigoplus_{i=1}^{n} \omega_{g}(f, \delta) .
$$

By direct computation we obtain

$$
\begin{aligned}
\omega_{g}(f, n \delta) & \leq g^{-1}\left(\sum_{i=1}^{n} g\left(\omega_{g}(f, \delta)\right)\right)=g^{-1}\left(n \cdot g\left(\omega_{g}(f, \delta)\right)\right) \\
& =g^{-1}\left(g g^{-1}(n) \cdot g\left(\omega_{g}(f, \delta)\right)\right)=g^{-1}(n) \odot \omega_{g}(f, \delta) .
\end{aligned}
$$


(vi) By using (ii) we have

$$
\omega_{g}(f, \lambda \delta) \leq \omega_{g}(f,([\lambda]+1) \delta)
$$

where $[\lambda]$ is the integer part of $\lambda$. Then by $(\mathrm{v})$ we get

$$
\omega_{g}(f, \lambda \delta) \leq g^{-1}([\lambda]+1) \odot \omega_{g}(f, \delta) .
$$

Since both $g^{-1}$ and $\odot$ are nondecreasing, finally we obtain

$$
\omega_{g}(f, \lambda \delta) \leq g^{-1}(\lambda+1) \odot \omega_{g}(f, \delta) .
$$

(vii) Let $x, y \in X$ be such that $d(x, y) \leq \delta$. Then, by the properties of the usual modulus of continuity, by taking into account that $g^{-1}$ is nondecreasing we have

$$
d_{g}(f(x), f(y))=g^{-1}|g f(x)-g f(y)| \leq g^{-1}(\omega(g f, \delta))
$$

and the inequality

$$
\omega_{g}(f, \delta) \leq g^{-1}(\omega(g f, \delta))
$$

follows. For the converse inequality let us start with

$$
|g f(x)-g f(y)|=g g^{-1}|g f(x)-g f(y)|=g\left(d_{g}(f(x), f(y)) .\right.
$$

By taking into account that $g$ is nondecreasing and the property in (i) of this Theorem we get

$$
|g f(x)-g f(y)| \leq g\left(\omega_{g}(f, \delta)\right)
$$

Taking supremum in this inequality we get

$$
\omega(g f, \delta) \leq g\left(\omega_{g}(f, \delta)\right)
$$

and since $g^{-1}$ is nondecreasing we obtain the required conclusion.

(viii) Let us suppose first that $f$ is continuous in the Euclidean distance. Then since $g f$ is also continuous and $g^{-1}$ is continuous at 0 and $g^{-1}(0)=0$, we obtain $\lim _{\delta \rightarrow 0} \omega_{g}(f, \delta)=\lim _{\delta \rightarrow 0} g^{-1}(\omega(g f, \delta))=0$. Conversely, let us suppose that $\lim _{\delta \rightarrow 0} \omega_{g}(f, \delta)=\lim _{\delta \rightarrow 0} g^{-1}(\omega(g f, \delta))=0$. Since $g^{-1}$ is continuous at 0 we get $\lim _{\delta \rightarrow 0} \omega(g f, \delta)=0$, which implies $g f$ is continuous. Since $g^{-1}$ is continuous, we obtain that $f$ is continuous and the proof is complete.

Further we obtain two properties for $d_{g}$ which will be used later.

Lemma 14 For any $a_{i}, b_{i} \in[0, \infty), i \in\{0, \ldots, n\}$ we have

$$
d_{g}\left(\bigoplus_{i=0}^{n} a_{i}, \bigoplus_{i=0}^{n} b_{i}\right) \leq \bigoplus_{i=0}^{n} d_{g}\left(a_{i}, b_{i}\right) .
$$


PROOF. By direct computation we have

$$
\begin{aligned}
d_{g}\left(\bigoplus_{i=0}^{n} a_{i}, \bigoplus_{i=0}^{n} b_{i}\right) & =g^{-1}\left|g\left(\bigoplus_{i=0}^{n} a_{i}\right)-g\left(\bigoplus_{i=0}^{n} b_{i}\right)\right| \\
& =g^{-1}\left|\sum_{i=0}^{n} g\left(a_{i}\right)-\sum_{i=0}^{n} g\left(b_{i}\right)\right| \\
& \leq g^{-1} \sum_{i=0}^{n} g g^{-1}\left|g\left(a_{i}\right)-g\left(b_{i}\right)\right| \\
& =g^{-1} \sum_{i=0}^{n} g d_{g}\left(a_{i}, b_{i}\right)=\bigoplus_{i=0}^{n} d_{g}\left(a_{i}, b_{i}\right) .
\end{aligned}
$$

Lemma 15 For any $x, y, z \in[0, \infty)$ we have

$$
d_{g}(x \odot y, x \odot z)=x \odot d_{g}(y, z) .
$$

PROOF. Indeed

$$
\begin{aligned}
d_{g}(x \odot y, x \odot z) & =g^{-1}|g(x) \cdot g(y)-g(x) \cdot g(z)| \\
& =g^{-1}\left(g(x) \cdot g g^{-1}(|g(y)-g(z)|)\right) \\
& =x \odot d_{g}(y, z)
\end{aligned}
$$

which completes the proof.

\subsection{Pseudo-linear approximation based on generated pseudo-operations}

Let $(X, d)$ be a compact metric space and let us consider a function $f: X \rightarrow$ $[0, \infty)$, continuous in the Euclidean distance, and $n \geq 1$ an arbitrary fixed natural number. Let $x_{i} \in X, i \in\{0, \ldots, n\}$ be fixed sampled data (i.e. we suppose that the values $f\left(x_{i}\right)$ are known). The idea of defining pseudo-linear approximation operators is very simple: we change the algebraic structure from the classical field of reals to that of an ordered semiring with generated pseudo-operations over the positive real numbers. The general discrete form of a pseudo-linear approximation is

$$
P_{n}(f, x)=\bigoplus_{i=0}^{n} K_{n}\left(x, x_{i}\right) \odot f\left(x_{i}\right),
$$

where $K_{n}\left(\cdot, x_{i}\right): X \rightarrow[0, \infty), i=0, \ldots, n$ are some given functions, which are continuous in the usual sense. 
Proposition 16 Let $C(X,[0, \infty))$ be the space of continuous functions $f$ : $X \rightarrow[0, \infty)$ endowed with the uniform $g$-distance (continuity of $f$ is understood in the Euclidean distance).

The operator $P_{n}: C(X,[0, \infty)) \rightarrow C(X,[0, \infty)), P_{n}(f, x)=\bigoplus_{i=0}^{n} K_{n}\left(x, x_{i}\right) \odot$ $f\left(x_{i}\right)$, is continuous and pseudo-linear, that is

$$
P_{n}\left(\alpha \odot f_{1} \oplus \beta \odot f_{2}, x\right)=\alpha \odot P_{n}\left(f_{1}, x\right) \oplus \beta \odot P_{n}\left(f_{2}, x\right) .
$$

PROOF. By using Lemmas 14 and 15 we have by direct computation

$$
d_{g}\left(P_{n}\left(f_{1}, x\right), P_{n}\left(f_{2}, x\right)\right) \leq \bigoplus_{i=0}^{n} K_{n}\left(x, x_{i}\right) \odot d_{g}\left(f_{1}\left(x_{i}\right), f_{2}\left(x_{i}\right)\right)
$$

and the continuity of $P_{n}$ in the uniform g-distance follows by the boudedness of $K_{n}\left(x, x_{i}\right)$ and the monotonicity of the pseudo-operations. The pseudo-linearity property is obvious.

The following Theorem provides a preliminary error estimate which will be helpful in obtaining the error estimates in the case of particular approximation operators.

Theorem 17 Let $f$ be as above, $n \geq 1$ and $K_{n}\left(\cdot, x_{i}\right): X \rightarrow[0,1]$ such that $\bigoplus_{i=0}^{n} K_{n}\left(x, x_{i}\right)=\mathbf{1}$, for any $x \in X$. Then for the approximation by $P_{n}(f, x)=$ $\bigoplus_{i=0}^{n} K_{n}\left(x, x_{i}\right) \odot f\left(x_{i}\right)$ we have the following error estimate

$$
d_{g}\left(P_{n}(f, x), f(x)\right) \leq \bigoplus_{i=0}^{n} K_{n}\left(x, x_{i}\right) \odot g^{-1}\left(m \cdot d\left(x, x_{i}\right)+1\right) \odot \omega_{g}\left(f, \frac{1}{m}\right),
$$

for any $x \in X$ and $m \geq 1$.

PROOF. Since $\bigoplus_{i=0}^{n} K_{n}\left(x, x_{i}\right)=\mathbf{1}$ we have

$$
d_{g}\left(P_{n}(f, x), f(x)\right)=d_{g}\left(\bigoplus_{i=0}^{n} K_{n}\left(x, x_{i}\right) \odot f\left(x_{i}\right), \bigoplus_{i=0}^{n} K_{n}\left(x, x_{i}\right) \odot f(x)\right) .
$$

By Lemma 14 we obtain

$$
d_{g}\left(P_{n}(f, x), f(x)\right) \leq \bigoplus_{i=0}^{n} d_{g}\left(K_{n}\left(x, x_{i}\right) \odot f\left(x_{i}\right), K_{n}\left(x, x_{i}\right) \odot f(x)\right)
$$


and by Lemma 15 we get

$$
d_{g}\left(P_{n}(f, x), f(x)\right) \leq \bigoplus_{i=0}^{n} K_{n}\left(x, x_{i}\right) \odot d_{g}\left(f\left(x_{i}\right), f(x)\right) .
$$

By the properties of the g-modulus of continuity and by taking into account that $\odot$ is nondecreasing, it follows that

$$
\begin{aligned}
d_{g}\left(P_{n}(f, x), f(x)\right) & \leq \bigoplus_{i=0}^{n} K_{n}\left(x, x_{i}\right) \odot \omega_{g}\left(f, d\left(x_{i}, x\right)\right) \\
& =\bigoplus_{i=0}^{n} K_{n}\left(x, x_{i}\right) \odot \omega_{g}\left(f, \frac{m \cdot d\left(x_{i}, x\right)}{m}\right) .
\end{aligned}
$$

Finally by Theorem 13, (vi) and the associativity of $\odot$, for any $m \in \mathbb{N}$ we obtain

$$
d_{g}\left(P_{n}(f, x), f(x)\right) \leq \bigoplus_{i=0}^{n} K_{n}\left(x, x_{i}\right) \odot g^{-1}\left(m \cdot d\left(x, x_{i}\right)+1\right) \odot \omega_{g}\left(f, \frac{1}{m}\right) .
$$

The next corollary gives sufficient conditions for the kernel functions in order to obtain Jackson-type estimate and approximation property of Weierstrass type in the g-distance $d_{g}$.

Corollary 18 Let $(X, d)$ and $f$ be as above and $n \geq 1$ be arbitrary. If $\oplus$ and $\odot$ are pseudo-operations given by a continuous nondecreasing generator and if the functions $K_{n}\left(\cdot, x_{i}\right), i=0, \ldots, n$ satisfy the conditions:

(i) $\bigoplus_{i=0}^{n} K_{n}\left(x, x_{i}\right)=\mathbf{1}$, for any $x \in X$;

(ii) There exists a constant $C$ and a set of points $x_{i} \in X, i \in\{0, \ldots, n\}$ satisfying $\bigoplus_{i=0}^{n} K_{n}\left(x, x_{i}\right) \odot g^{-1}\left(n \cdot d\left(x, x_{i}\right)+1\right) \leq C$.

Then for the approximation

$$
P_{n}(f, x)=\bigoplus_{i=0}^{n} K_{n}\left(x, x_{i}\right) \odot f\left(x_{i}\right),
$$

we have the Jackson-type error estimate

$$
d_{g}\left(P_{n}(f, x), f(x)\right) \leq C \odot \omega_{g}\left(f, \frac{1}{n}\right)
$$

and $P_{n}(f, x)$ converges $g$-uniformly to $f(x)$ on $X$. 
The condition (i) is natural. If we consider condition (ii), however it is not easy to see which approximation operators fulfill it, we will give in the followings an example of such approximation operator. The algebraic structure in this example will be a special case of ordered semiring with generated pseudooperations, obtained via a particular family of generators.

In this sense we let $g:[0, \infty) \rightarrow[0, \infty), g(x)=x^{a}$, where $a>0$.

Firstly we observe that in this case

$$
x \oplus y=\left(x^{a}+y^{a}\right)^{\frac{1}{a}}
$$

and that

$$
x \odot y=x \cdot y
$$

i.e., the $\odot$ operation is the classical multiplication. For simplicity we will define and study the Shepard-type pseudo-linear approximation operator only in this simple case, however it can be defined similarly for other pseudo-operations as well. Surely the study of those cases is subject of further research.

Next we define Shepard-type approximation operators based on the pseudooperations given above. We consider $f:[0,1] \rightarrow[0, \infty)$ continuous in the Euclidean sense and equally spaced $\operatorname{knots} x_{i}=\frac{i}{n}, i=0, \ldots, n$. Also, let $a>0$ denote the parameter of the pseudo-addition and $\lambda>0$ be such that $a \cdot \lambda>2$.

The $\oplus$-product Shepard kernel is

$$
K_{n}^{\lambda}\left(x, x_{i}\right)=\frac{\frac{1}{\left|x-x_{i}\right|^{\lambda}}}{\bigoplus_{j=0}^{n} \frac{1}{\left|x-x_{j}\right|^{\lambda}}} \text {, if } x \notin\left\{x_{0}, . ., x_{n}\right\}
$$

and $K_{n}^{\lambda}\left(x_{j}, x_{i}\right)=\delta_{i j}, i, j=0, \ldots, n$. It is easy to check that the functions $K_{n}^{\lambda}\left(\cdot, x_{i}\right), i=0, \ldots, n$ are continuous in the Euclidean distance. The $\oplus$-product Shepard-Balázs operator is given by

$$
S h_{n}^{\lambda}(f, x)=\bigoplus_{i=0}^{n}\left(\frac{\frac{1}{\left|x-x_{i}\right|^{\lambda}}}{\bigoplus_{j=0}^{n} \frac{1}{\left|x-x_{j}\right|^{\lambda}}} f\left(x_{i}\right)\right) \text {, if } x \notin\left\{x_{0}, . ., x_{n}\right\}
$$

and $S h_{n}^{\lambda}\left(f, x_{i}\right)=f\left(x_{i}\right), i=0, \ldots, n$. It is easy to check that $S h_{n}^{\lambda}(f, \cdot)$ is continuous in the usual sense.

In the next theorem we obtain the error bound for the approximation by this operator on the unit interval in terms of the g-distance. Here we will use some properties related to the classical Shepard-Balázs operator. 
Theorem 19 Let $f:[0,1] \rightarrow[0, \infty)$ be continuous in the Euclidean sense $n \geq 1$ and $x_{i}=\frac{i}{n}, i \in\{0, \ldots, n\}$ Also, let $a>0$ denote the parameter of the pseudo-addition in (13) and $\lambda>0$ be such that $a \cdot \lambda>2$. For the error estimate in approximation by the $\oplus$-product Shepard operator in (14) we have the following Jackson-type estimate

$$
d_{g}\left(S h_{n}^{\lambda}(f, x), f(x)\right) \leq C \cdot \omega_{g}\left(f, \frac{1}{n}\right), \forall x \in[0,1],
$$

where $C$ is some constant which does not depend on $n$.

PROOF. We observe that

$$
K_{n}^{\lambda}\left(x, x_{i}\right)=\frac{\frac{1}{\left|x-x_{i}\right|^{\lambda}}}{\bigoplus_{j=0}^{n} \frac{1}{\left|x-x_{j}\right|^{\lambda}}}=\frac{\frac{1}{\left|x-x_{i}\right|^{\lambda}}}{\left(\sum_{j=0}^{n} \frac{1}{\left|x-x_{j}\right|^{\lambda a}}\right)^{\frac{1}{a}}} .
$$

By Corollary 18, it is enough to estimate the quantity

$$
E_{n}^{\lambda}(x)=\bigoplus_{i=0}^{n} K_{n}^{\lambda}\left(x, x_{i}\right) \odot g^{-1}\left(n \cdot\left|x-x_{i}\right|+1\right) .
$$

By taking into account the definitions of the operation $\oplus$ and the definition of $g$ and $g^{-1}$ we get

$$
E_{n}^{\lambda}(x)=\left(\sum_{i=0}^{n} K_{n}^{\lambda}\left(x, x_{i}\right)^{a} \cdot\left(n \cdot\left|x-x_{i}\right|+1\right)\right)^{\frac{1}{a}} .
$$

By direct computation we obtain

$$
E_{n}^{\lambda}(x)=\left(\sum_{i=0}^{n} \frac{\frac{1}{\left|x-x_{i}\right|{ }^{\alpha}}}{\sum_{j=0}^{n} \frac{1}{\left|x-x_{j}\right|^{\lambda a}}} \cdot\left(n \cdot\left|x-x_{i}\right|+1\right)\right)^{\frac{1}{a}}
$$

and finally

$$
E_{n}^{\lambda}(x)=\left(\frac{n \sum_{i=0}^{n} \frac{1}{\left|x-x_{i}\right|^{a \lambda-1}}}{\sum_{j=0}^{n} \frac{1}{\left|x-x_{j}\right|^{a \lambda}}}+1\right)^{\frac{1}{a}} .
$$

Now, let us recall that

$$
\left(\sum_{i=0}^{n} \frac{1}{\left|x-x_{i}\right|^{\gamma}}\right)^{-1}=O\left(n^{-\gamma}\right)
$$


for any $\gamma>1$ (see [27], [31], [32], [28]).

Then since $a \lambda-1>1$ we have

$$
E_{n}^{\lambda}(x)=\left(\frac{n \cdot O\left(n^{-a \lambda}\right)}{O\left(n^{1-a \lambda}\right)}+1\right)^{\frac{1}{a}}=O(1),
$$

i.e., there exists a constant $C$ such that

$$
d_{g}\left(S h_{n}^{\lambda}(f, x), f(x)\right) \leq C \cdot \omega_{g}\left(f, \frac{1}{n}\right)
$$

and the proof is complete.

The following Corollary shows that the error estimate in terms of the gdistance obtained in the previous Theorem implies the g-uniform convergence of $S h_{n}^{\lambda}(f, x)$ to $f(x)$. Let us mention that the g-uniform convergence implies the pointwise convergence in the Euclidean distance.

Corollary 20 Let $f:[0,1] \rightarrow[0, \infty)$ be continuous in the Euclidean sense, $a, \lambda$ as above and the pseudo-linear Shepard operator given by (14). Then $S h_{n}^{\lambda}(f, x)$ converges g-uniformly to $f(x)$.

PROOF. It is obvious by the preceding Theorem 19 and by Theorem 13, (viii).

\section{Concluding remarks}

We have introduced new particular approximation operators defined based on three types of ordered semiring structures. We have obtained general error bounds, Weierstrass-type approximation theorems and particular error bounds in some particular cases for all these operators. Let us remark that the error estimates for the approximation operators defined in this paper are of Jackson type i.e., $O\left(\omega\left(f, \frac{1}{n}\right)\right)$ and $O\left(\omega_{g}\left(f, \frac{1}{n}\right)\right)$ respectively, so these have approximation properties which are similar to the classical Jackson, Shepard or spline approximation operators (see e.g. [5]), and in some cases they are more general or "outperform" them from the theoretical point of view.

It is easy to see that the results proposed in the present paper extend classical approximation theory into a new direction. As far as the computational complexity is concerned, if we regard max-product and max-min operations 
they are less expensive. If we consider generated pseudo-operations the computational complexity depends on the expression of the generator. This issue would certainly be worth investigating.

Approximation Theory is one of the basic tools of Image Processing. So if we provide an approximation method then it is immediately interpreted as an image compression method. So we propose as a further research topic the application of pseudo-linear approximation operators in image compression. Another promising research topic is the use of the proposed approximations for noise reduction in images.

Acknowledgement. The authors would like to express their thanks to the Editors in Chief, the Area Editor and two anonymous referees for their comments and suggestions which improved the paper.

Moreover, the third author would like to acknowledge support of the project MSM6198898701 of the MŠMT ČR.

\section{References}

[1] J. Aczél, Lectures on Functional Equations and their Applications, Academic Press, New York, 1966.

[2] G. A. Anastassiou, Rate of convergence of fuzzy neural network operators, univariate case, J. Fuzzy Math. 10, No. 3(2002), 755-780.

[3] G. A. Anastassiou, S.G. Gal, Approximation Theory: Moduli of Continuity and Global Smoothness Preservation, Birkhäuser, Boston-Basel-Berlin, 2000.

[4] B. Bede, H. Nobuhara, J. Fodor, K. Hirota, Max-Product Shepard Approximation Operators, Journal of Advanced Computational Intelligence and Intelligent Informatics, 10 (2006), 494-497.

[5] R.A. DeVore, G.G. Lorentz, Constructive Approximation, Polynomials and Splines Approximation, Springer-Verlag, Berlin, Heidelberg, 1993.

[6] R.A. DeVore, V.N. Temlyakov, Nonlinear approximation in finite-dimensional spaces, J. Complexity, 13 (1997), 489-508.

[7] W.D. Fisher, On grouping for maximum homogeneity, J. Amer. Statist. Assoc. 53 (1958) 789-798.

[8] M. Gondran, M. Minoux, Dioïds and semirings: Links to fuzzy sets and other applications, Fuzzy Sets and Systems 158 (2007) 1273 - 1294.

[9] K. Hirota, W. Pedrycz, Data compression with fuzzy relational equations, Fuzzy Sets and Systems, 126(2002), 325-335. 
[10] L.T. Kóczy, K. Hirota, Approximate reasoning by linear rule interpolation and general approximation, International Journal of Approximate Reasoning 9(1993), 197-225.

[11] V.P. Maslov, S.N.Samborskii, Idempotent Analysis, Adv. Soc. Math. 13, Amer. Math. Soc. Providence, RI, 1992.

[12] G. Mastroianni and J. Szabados, Bala zs Shepard Operators on Infinite Intervals, II, Journal of Approximation Theory 90(1997) 1-8.

[13] R. Mesiar, J. Rybárik, Pan-operations structure, Fuzzy Sets and Systems, 74(1995), 365-369.

[14] M. Minoux, Polynomial approximation schemes and exact algorithms for optimum curve segmentation problems, Discrete Applied Mathematics 144 (2004) 158-172.

[15] J. Musielak, Nonlinear Approximation in some modular function spaces I, Math. Japonica, 38(1993), 83-90.

[16] H. Nobuhara, W. Pedrycz, K. Hirota, Fast solving method of fuzzy relational equations and its applications to lossy image compression/reconstruction, IEEE Transactions on Fuzzy systems, 3(2000), 325-334.

[17] E. Pap, K. Jegdić, Pseudo-analysis and its application in railway routing, Fuzzy Sets and Systems, 116(2000), 103-118.

[18] E. Pap, I. Stajner-Papuga, A limit theorem for triangle functions, Fuzzy Sets and Systems, 157(2006), 292-307.

[19] E. Pap, Pseudo-additive measures and their applications, in Handbook of Measure Theory (E. Pap, ed.), Elsevier Science B.V., 2002.

[20] E. Pap, Applications of the generated pseudo-analysis to nonlinear partial differential equations, Contemporary Mathematics, 377(2005), 239-259.

[21] I. Perfilieva, Fuzzy function as an approximate solution to a system of fuzzy relation equations, Fuzzy Sets and Systems, 147(2004), 363-383.

[22] I. Perfilieva, Fuzzy Transforms, in Transactions on Rough Sets II (J.F. Peters et al. Eds.) LNCS 3135 (2004), 63-81.

[23] B. Schweizer, A. Sklar, Probabilistic Metric Spaces. North-Holland, Amsterdam 1983.

[24] D. Shepard, A two-dimensional interpolation function for irregularly spaced data, Proc. 1968 ACM National Conference, 517-524.

[25] G. Somorjai, On a saturation problem, Acta Math. Acad. Sci. Hungar. 32(1978) 377-381.

[26] J. Szabados, On a problem of R. DeVore, Acta Math. Hungar. 27 (1-2)(1976) 219-223. 
[27] J. Szabados, Direct and converse approximation theorems for the Shepard operator, Approximation Theory and Applications 7(1991), 63-76.

[28] D. Tikk, Notes on the approximation rate of fuzzy KH interpolators, Fuzzy Sets and Systems 138(2003), 441-453.

[29] E. Trillas, C. Alsina, Introduccion a los Espacios Metricos Generalizados.Fund. J. March. Serie Universitaria 49(1978), Madrid.

[30] L. Valverde, On the structure of F-indistinguishability operators, Fuzzy Sets and Systems 17(1985) 313-328.

[31] P. Vértesi, Saturation of the Shepard operator, Acta Math. Hungar. 72(1996) 307-317.

[32] W. Xiao, S.P. Zhou, A Jackson-type estimate for Shepard operators in $L^{p}$ spaces, for $p \geq 1$, Acta Mathematica Hungarica, 95(2002), 217-224.

[33] X. Zhou, The saturation class of Shepard operators, Acta Mathematica Hungarica, 80(1998), 293-310. 